\title{
La evaluación de la calidad de las noticias en la prensa vasca, en comparación con la prensa europea de referencia
}

\author{
Alazne AIESTARAN \\ Universidad del País Vasco - UPV/EHU \\ alazne.aiestaran@ehu.es \\ Antxoka AgIRRe \\ Universidad del País Vasco - UPV/EHU \\ antxoka.agirre@ehu.es \\ Beatriz ZABALONDO \\ Universidad del País Vasco - UPV/EHU \\ beatriz.zabalondo@ehu.es \\ Txema RAMíREZ DE LA PISCINA \\ Universidad del País Vasco - UPV/EHU \\ txema.ramirezdelapiscina@ehu.es
}

Recibido: 5 de diciembre de 2014

Aceptado: 8 de mayo de 2015

\section{Resumen}

Este trabajo recoge las principales conclusiones de una investigación cuantitativa y cualitativa sobre la evolución de la calidad de las noticias publicadas en las versiones impresas y electrónicas del diario Euskaldunon Egunkaria nacido en 1990 y su directo sucesor Berria (nacido en 2003), el histórico semanario Argia surgido en 1919 y Goiena, nacido como Goienkaria en 2000, una publicación bisemanal que se edita en la comarca del Alto Deba (Gipuzkoa), y las compara con cinco diarios referentes en Europa -Financial Times, Le Monde, Frankfurter Allgemeine Zeitung, Corriere della Sera, y El País-. El equipo de investigación ha utilizado un método de análisis fundamentado en el concepto de "media performance" desarrollado por Denis McQuail (1992). Las conclusiones reflejan una tendencia decreciente de la calidad en la prensa y en la europea.

Palabras clave: Periodismo de calidad, medios vascos, lenguas minorizadas, calidad de las noticias, futuro del periodismo.

\section{Evaluation of the news quality of the Basque press compared with the European reference press}

\begin{abstract}
This work sets out the main conclusions of a quantitative and qualitative study of the evolution in the quality of news items published in the printed and digital versions of the daily newspaper Euskaldunon Egunkaria, born in 1990 and its direct successor Berria (born in 2003), the long-running weekly publication Argia appearing in 1919 and Goiena, born as Goienkaria in 2000, a bi-weekly publication which is published in the area of the Alto Deba (Gipuzkoa), and compares them with five daily newspapers referent in Europe: Financial Times, Le Monde, Frankfurter Allgemeine Zeitung, Corriere della Sera, and El Pais. The research team has used an analysis method based on the concept of "media performance" developed by Denis McQuail (1992). The conclusions reflect a downward trend in quality both in the Basque and European press.
\end{abstract}

Keywords: Quality journalism, Basque Media, minority languages, news quality, future of journalism. 


\section{Referencia normalizada}

AIESTARÁN, Alazne; AGIRRE, Antxoca ; ZABALONDO, Beatriz; y RAMÍREZ DE LA PISCINA, Txema (2015): "La evaluación de la calidad de las noticias en la prensa vasca, en comparación con la prensa europea de referencia". Estudios sobre el Mensaje Periodístico. Especial noviembre "Periodismo e información de calidad", págs.: 47-62. Madrid, Servicio de Publicaciones de la Universidad Complutense.

Sumario: 1.Introducción; 1.1. Los medios de comunicación en euskara en la actualidad; 1.2. Investigaciones recientes. 2. Objetivos, hipótesis y preguntas de investigación (RQ). 3. Metodología. 4. Resultados; 4.1. Argia, análisis diacrónico; 4.1.1. Argia, análisis sincrónico; 4.2. Berria, análisis diacrónico; 4.2.1. Berria, análisis sincrónico; 4.3. Goiena, análisis diacrónico; 4.3.1. Goiena, análisis sincrónico; 4.4. Comparación de la prensa vasca con la prensa europea de referencia; 4.4.1. Comparativa de los promedios; 4.4.2. Índices distribuidos por niveles; 4.4.3. Diversidad; 4.4.4. El salto a internet. 5. Conclusiones. 6. Referencias bibliográficas.

\section{Introducción}

\subsection{Los medios de comunicación en euskara}

Pese a que el euskara es una de las lenguas más antiguas de Europa y sin Estado propio, su regulación fue tardía respecto a las lenguas que convivían a su alrededor. Ello justifica, entre otras razones, el retraso de la aparición de la prensa vasca y el número de cabeceras, en relación a la prensa francesa o española. El punto de partida lo marcó en 1848 Uscal-Herrico Gaseta, primer periódico íntegramente en lengua vasca (Díaz Noci, 2012: 83). A partir de ese momento y hasta llegar a nuestros días la prensa vasca ha estado marcada desde sus orígenes por problemas económicos. Ha subsistido en gran medida gracias al trabajo voluntario de muchas personas que creían y creen en el euskara, y ven imprescindible que esté presente a través de los medios de comunicación.

En Euskal Herria en 2009 se editaban en torno a 110 medios en lengua vasca, pero teniendo en cuenta que en los últimos años han desaparecido varios medios a causa de la crisis económica, hoy en día la cifra se ha reducido "un $8 \%$, lo que significa que ahora, a principios de 2014, hay cerca de 10 medios menos que en 2009. El sector que más sufrió fue el de la prensa" (Zabaleta et al., 2014: 108).

Salvo el ente público EITB, el resto de los medios pertenecen al sector privado. Dentro del sector privado cuantitativamente tienen un peso muy importante los medios locales en euskara, fenómeno que surge a finales de los 80 y se expande con fuerza en la siguiente década. Eneko Bidegain, profesor e investigador de la Universidad de Mondragón, contabilizó 86 medios locales en el año 2013 clasificados por periodicidad en: 48 revistas, semanales o diarios, 20 radios, 7 televisiones y 9 publicaciones de internet. De los 86 medios locales, 2 pertenecen a Álava, 14 a Vizcaya, 42 a Guipúzcoa, 3 a Lapurdi, 10 a Navarra, 3 a la Baja Navarra y 2 a Zuberoa (VV.AA., 2013: 21).

\subsection{Investigaciones recientes}

Este trabajo parte de investigaciones anteriores tanto relativas a los medios en euskara como a las que han tenido como objetivo la calidad de la información.

McQuail (1992), que desarrolló el concepto de media performance ha sido una referencia imprescindible en esta investigación. Por su parte, los autores escandinavos han sido pioneros con sus estudios sobre la calidad y en la creación del concepto de informatividad mientras que en Estados Unidos, la perspectiva comercial definió la direc- 
ción de las investigaciones. La comunidad científica alemana, sin embargo, articuló el marco teórico del debate sobre la calidad, junto con algunas propuestas metodológicas.

Aunque académicos y profesionales admitan estar preocupados por la calidad de las noticias que se ofrecen a diario en los medios, no son muy abundantes los estudios rigurosos que se han realizado en esa línea. Citemos, a título meramente ilustrativo los trabajos realizados en UCLA por Ryan Ozimek (1998), John Zaller (1999) y Robert Butche (2008), o por Esther Thorson (2001) de la universidad de Missouri, Ivor Shapiro de la Universidad de Wyoming (2004) y Gabriel Michi en Argentina (2010). Asimismo, entidades como The Berkman Center for Internet \& Society de la Universidad de Harvard o el European Journalism Centre de Amsterdam también han demostrado estar preocupados por la calidad de la información. En algunos países latinoamericanos la investigación en torno a la calidad se ha centrado en la responsabilidad social y la calidad democrática (Gómez Mompart y Palau, 2013: 22).

El interés y la reflexión en torno a la calidad de los contenidos en los medios de comunicación en euskara es una cuestión que lleva presente una década entre los profesionales, académicos e instituciones vascas. El equipo encargado de organizar el Primer Congreso de Periodismo en Euskara, que se celebró entre los días 9 y 11 de noviembre de 2004 en el Palacio Euskalduna de Bilbao, publicó en 2005 un libro con las reflexiones y las conclusiones más importantes obtenidas en el congreso donde se hacía alusión directa a la calidad del periodismo en euskara: "Si el periodismo en euskara quiere avanzar, lo hará porque garantizará unos estándares mínimos de calidad" (VV.AA., 2005: 447).

Son varios los autores que han analizado los medios editados íntegramente en euskara: Camacho, 1999, Arana et al. 2000, Zuberogoitia 2003, Aiestaran 2007 y 2011, Zabaleta 2010, Zabalondo 2011, Díaz Noci 2012, Bidegain 2013. Sin embargo, ninguno de ellos ha estudiado la calidad de la información. En ese sentido, la aportación más reciente es la tesis doctoral de Maria Gonzalez Gorosarri presentada en 2011 en la Universidad del País Vasco y que ha consistido en desarrollar la metodología para medir la calidad de las noticias, unificando los criterios válidamente probados por trabajos anteriores.

\section{Objetivos, hipótesis y preguntas de investigación (RQ)}

El principal objetivo de este estudio es analizar la evolución de la calidad de las noticias publicadas por los principales medios en lengua vasca -tanto en su versión impresa como electrónica- en un período comprendido entre 2001 y 2013 y compararlas con cinco diarios de referencia europeos (El País (EP), Le Monde (LM), Frankfurter Allgemeine Zeitung (FAZ), Corriere della Sera (CDS) y Financial Times (FT).

El equipo de investigación considera que el periodismo desarrollado en una lengua minorizada como es el euskara, puede obtener unos resultados de calidad equiparables a los estándares de calidad de los diarios referentes en Europa. Del mismo modo, creemos que la comparación entre la prensa vasca y europea puede servir para conocer los puntos flacos del periodismo producido en una lengua de reducida difusión, así como para conocer si el periodismo realizado en lenguas minorizadas pueda hacer aportaciones en el ámbito de la calidad al periodismo elaborado en las lenguas dominantes o más fuertes. 
Para conseguir dar respuesta y esclarecer las cuestiones propuestas, el equipo plantea las siguientes Hipótesis $(\mathrm{H})$ y preguntas de investigación o research questions (RQ):

H1.El desarrollo tecnológico al que la prensa escrita está sometida en el siglo XXI ha ocasionado una aceleración en los procesos de selección y elaboración de la información y ello puede influir en la calidad de la información que se ofrece.

Research Questions asociadas a la hipótesis:

$R Q 1$ - ¿Cuál ha sido la evolución de la calidad de la información de la prensa vasca entre los años 2001 y 2012?

$R Q 2-$ ¿Se aprecian diferencias notables entre las publicaciones vascas analizadas?

$R Q 3-$ ¿El formato de la información analizada (la principal noticia del día aparecida en portada, la noticia de gran formato que desarrollaba la anterior en páginas interiores, y una noticia considerada de segundo orden, publicada a una o, a lo sumo, dos columnas) influye en el índice de calidad?

H2. La digitalización de los contenidos y la inmediatez que exigen las ediciones digitales han acelerado aún más el proceso de elección y el proceso de elaboración. Esa situación puede repercutir en la calidad de la información que se ofrece.

Research Questions asociadas a la hipótesis:

$R Q 4-$ ¿Cuáles son las diferencias que existen entre los diarios analizados?

RQ5- ¿Los resultados del análisis sincrónico del 2013 y los datos del análisis diacrónico de 2001-2012 son coherentes entre sí?

H3. El nivel del periodismo elaborado en lenguas minorizadas como el euskara puede alcanzar un nivel digno frente al periodismo producido en lenguas dominantes. Este tipo de comparaciones resultan de gran interés para las lenguas minorizadas, dado que muestran los aspectos a mejorar. Del mismo modo, el periodismo en lenguas minorizadas puede aportar aspectos positivos al campo del periodismo en lenguas mayoritarias.

Research Questions asociadas a la hipótesis:

RQ6- ¿Qué nivel de calidad ofrecen las publicaciones vascas en comparación con los diarios de referencia europeos?

RQ7- ¿Qué carencias se aprecian en la calidad del periodismo en euskara?

RQ8- ¿En algún aspecto el periodismo elaborado en euskara supera la calidad del periodismo que se desarrolla en lenguas mayoritarias?

\section{Metodología}

El equipo de investigación que ha llevado a cabo este estudio ha utilizado una metodología propia basada en las contribuciones más importantes de la literatura germana y anglosajona de los últimos 10 años (Bucher y Altmerppen, 2003; Maurer, 2005; Vehlow, 2006).

La metodología establecida ha permitido al equipo medir parámetros cuantitativos y cualitativos con rigurosidad, tanto en las ediciones impresas como en las ediciones digitales de las publicaciones. El método se ajusta estrictamente a piezas informativas publicadas por la prensa y pertenecientes a géneros informativos, excluyéndose por tanto textos interpretativos como el análisis, el reportaje, la crónica, la entrevista o las columnas de opinión. Los parámetros que se citan a continuación 
están sujetos a discusión. El equipo ha pretendido objetivarlos al máximo, sabiendo que ello implica ciertos riesgos y estar sometido a la crítica. Pese a ello se ha creído que era absolutamente necesario realizar este esfuerzo (Ramirez de la Piscina et al., 2014).

En el primer apartado se incluyen las variables relacionadas con la calidad del formato (aspectos técnicos, fallos estéticos, funcionales, lapsus clavis). Todas y cada una de las variables mencionadas se contabilizan y se recogen para las conclusiones finales.

En la segunda parte se mide el índice de calidad en base a tres niveles de análisis a los que otorgamos un valor máximo: proceso de selección $(2,5)$, elaboración $(5)$ y aportación social $(2,5)$. La suma de los tres niveles es de 10 puntos. Cada uno de esos niveles contiene una serie de variables que se detallan a continuación:

Tabla 1. Fuente: Grupo de Investigación HGH

\begin{tabular}{|c|c|}
\hline INDICE DE CALIDAD & \\
\hline \multicolumn{2}{|l|}{ SELECCIÓN } \\
\hline Cita del origen de la información & Mencionado $(0,5)$; Indirectamente $(0,25)$; No Mencionado $(0)$ \\
\hline Carácter de las fuentes & Documentos $(0,5)$; Expertos $(0,4)$; Identficadas $(0,3)$; Indirectamente $(0,1)$, No mencionadas $(0)$ \\
\hline Factualidad & Suceso $(0,5)$; Declaraciones $(0,25)$; Anuncio $(0)$ \\
\hline Actualidad & $\begin{array}{l}\text { Digtales: Las dos útimas horas }(0,5) \text {; Digitales: "Ayer", "hoy", "manana" }(0,4) \text {; Prensa: "Ayer", "hoy", } \\
\text { "mañana" }(0,5) ; 2-7 \text { dias }(0,25) \text {, Más de una semana }(0,1) \text {; Sin fecha }(0)\end{array}$ \\
\hline Noticiabilidad & Consecuencias sociales $(0,5)$, Consecuencias personales $(0,25)$, Pseudevento $(0)$ \\
\hline \multicolumn{2}{|l|}{ ELABORACIÓN } \\
\hline Exactitud & Resume / Adelanta la noticia (1); Destaca solo una parte/carateristica $(0,5)$; Es ambiguo/engañoso (0) \\
\hline Profundidad & $7 \mathrm{~W}(1), 5-6 \mathrm{~W}(0,5) ; 4 \mathrm{~W}(0)$ \\
\hline Número de perspectivas & 3 perspectivas (1); 2 perspectivas $(0,5) ; 1$ perspectiva, razonada $(0,25) ; 1$ perspectiva, $\sin$ razonar $(0)$ \\
\hline Elementos informativos complementarios & Muy significativos $(4-5 \mathrm{~W})(0.5)$; Significativos $(2-3 \mathrm{~W})(0.25)$; Relleno (1W) (0); No coinciden $(-0.25)$ \\
\hline Aspecto artistico & $\begin{array}{l}\text { Muy elaborado }(0,5) \text {; Bastante elaborado }(0,25) \text {, Sencillo }(0,1) \text {; No tiene elementos adicionales }(0) \text {; Fallo } \\
\text { leve }(-0,25) \text {; Fallo grave }(-0,5) \text {; Fallo muy grave }(-0,75)\end{array}$ \\
\hline $\begin{aligned} \text { Lenguaje: Estilo periodistico } \\
\end{aligned}$ & Claro y conciso (1); No se entiende de una vez $(0)$; No profesional $(-0,5)$; Mediocre $(-1)$ \\
\hline \multirow{2}{*}{\multicolumn{2}{|c|}{$\begin{array}{l}\text { Errores: tifular, antetitular, subtitular }(-1) \\
\text { Errores ventanas, pie de foto ladillo }(-0,5)\end{array}$}} \\
\hline \multirow{2}{*}{\multicolumn{2}{|c|}{$\begin{array}{l}\text { Errores: ventanas, pie de foto, ladillo }(-0,5) \\
\text { Errores: texto }(-0,25)\end{array}$}} \\
\hline & \\
\hline \multicolumn{2}{|l|}{ APORTACIÓN SOCIAL } \\
\hline Control al poder & Controla al poder $(0,5)$; No controla al poder $(0)$; Asimila el discurso del poder $(-0,5)$ \\
\hline Fomento del debate social & Fomenta el debate $(0,5)$, No fomenta el debate $(0)$; Divide, rompe $(-0,5)$ \\
\hline Respeto a la dignidad humana & Garantiza la dignidad $(0.5)$; No garantiza la dignidad $(-0,5)$; Ni una ni otra $(0)$ \\
\hline Referencias culturales & Cultura local o mundial $(0,5)$; Sin referencia cultural alguna $(0)$; Cultura asimiladora $(-0,5)$ \\
\hline Combate la marginación & Combate la marginación $(0,5)$; No combate la marginación $(0)$; Insulta, desprecia $(-0,5)$ \\
\hline \multirow{2}{*}{\multicolumn{2}{|c|}{$\begin{array}{l}\text { Selección ( } 2,5 \text { puntos) } \\
\text { Elaboración (5 puntos) } \\
\text { Aportación social ( } 2,5 \text { puntos) }\end{array}$}} \\
\hline & \\
\hline TOTAL & \\
\hline
\end{tabular}

El método utilizado está recogido en un libro de códigos creado por el grupo investigador con el objetivo de establecer los acuerdos básicos sobre los que se asienta el método de medición de la calidad de las noticias (Ramirez de la Piscina et al., 2014). Mediante la metodología fijada se han analizado cinco diarios de referencia en Europa: El País (EP), Le Monde (LM), Frankfurter Allgemeine Zeitung (FAZ), Corriere de la Sera (CDS) y Financial Times (FT). Se ha llevado a cabo un análisis diacrónico de la evolución de la calidad en los diarios impresos entre el 1 de enero de 2001 y el 31 de diciembre de 2012. Para ello, nos hemos basado en la técnica de la semana compuesta, técnica reconocida y probada por varios autores entre los que destacan Riffe, Aust \& Lacy, Stempel (Zabaleta, 1997, pp. 199-203). Así, se analizaron 84 ediciones de cada diario y tres ítems por cada edición (la noticia principal de la portada, la noticia desarrollada en el interior que aparece en portada y una noticia de segunda categoría que aparece publicada a una o dos columnas también en el interior). En total, el número de noticias analizadas fue de 1.137 para los cinco diarios (252 de El País y Le Monde, 
224 de Frankfurter Allgemeine Zeitung, 219 Corriere de la Sera y 190 de Financial Times). En una segunda fase se analizaron las ediciones digitales de los diarios en el año 2013 y para ello se utilizó la técnica de la semana continua. Se examinaron tres semanas continuas: una del 23 al 29 de enero, otra del 6 al 12 de febrero y la tercera del 13 al 19 de marzo. Primero se analizó la noticia más importante de la edición digital y posteriormente al día siguiente se examinó esa misma noticia en la edición impresa. La muestra en este caso estuvo formada por 210 ítems (Ramirez de la Piscina et al., 2014).

Por último, se compararon los resultados obtenidos en 2013 con los recopilados en el análisis diacrónico (2001-2012), siempre que las variables permitiesen la comparación.

Hemos aplicado la misma técnica para el muestreo y el mismo método para medir la calidad de las noticias de las siguientes publicaciones en euskara: Argia publicación decana de la prensa vasca que nace en 1919 (84 ítems), Berria (164 ítems) sucesor directo en 2003 del clausurado ese mismo año Euskaldunon Egunkaria, y Goiena (240 ítems), referente de la prensa local en euskara creado en 2000 con el nombre de Goienkaria.

488 ítems componen la muestra del análisis diacrónico. Asimismo, con estas tres publicaciones se procedió al análisis de las ediciones digitales durante tres semanas de enero, febrero y marzo de 2013. En este caso se analizaron un total de 570 ítems.

\section{Resultados}

\subsection{Argia, análisis diacrónico}

Argia es una publicación semanal de 52 páginas que se distribuye en toda la geografía vasca, a saber, en la Comunidad Autónoma Vasca, Comunidad Foral de Navarra y el País Vasco del Norte. Debido a su periodicidad semanal se ha tenido que ajustar la técnica de la semana compuesta. En esta revista semanal solo se han analizado las noticias de gran formato, una por cada ejemplar establecido en el día correspondiente a la muestra.

Los textos informativos de Argia muestran un nivel de calidad muy alto. El promedio (pm) de la prensa de referencia en Europa (EuP) es de 5,39, el promedio de la prensa vasca (Euskal Prentsa, EkP) es de un 5,07 y la media de Argia es de un 6,23. Como ha quedado señalado, en el caso de Argia, por su formato y periodicidad semanal, sólo se ha analizado la noticia más importante. Esa es la razón principal, a nuestro juicio, que explica la puntuación alcanzada por Argia. Cabe subrayar que en el resto de los diarios analizados es la noticia de primera desarrollada en el interior, la que mejores resultados de calidad ha obtenido.

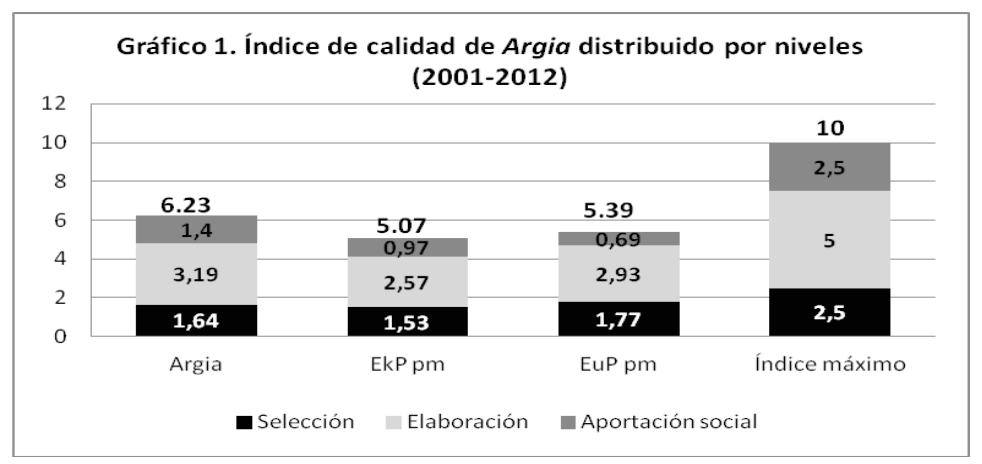

Fuente: Grupo de Investigación HGH 
En el apartado relativo al proceso de selección de la información, Argia consigue una puntuación de 1,64 de un total de 2,5. Ese valor está por encima del promedio de la prensa vasca y por debajo del promedio europeo. Debido a la periodicidad semanal, su punto débil es la actualidad $(0,11$ de 0,5$)$ y su aspecto más fuerte es la noticiabilidad $(0,48$ de 0,50$)$. En lo que se refiere a los principios de transparencia informativa, el trabajo de Argia es aceptable, ya que tanto en la mención del origen de la información como en la especificación de la identidad de las fuentes obtiene un 0,3 de un total de 0,5 . En la variable de la factualidad alcanza un valor alto $(0,45)$, porque son los hechos la base de sus informaciones y no las declaraciones o las especulaciones.

En la elaboración de contenidos recibe una mejor nota, puesto que elabora las informaciones con exactitud, en profundidad, incluyendo diversas perspectivas y con un estilo periodístico claro y conciso $(0,98$ de un máximo de 1$)$. Comete pocos errores, tanto en los elementos de titulación como en el texto (un promedio de - $-0,01$ de penalización en ambas variables). En el apartado de la elaboración sólo Frankfurter Allgemeine Zeitung $(3,44)$ se sitúa por encima de Argia.

En el bloque de aportación social, Argia consigue los mejores resultados entre todos los diarios analizados. El control al poder, característica propia de todo medio y el fomento del debate social son los aspectos más destacados de este apartado. De modo que se puede interpretar que Argia elabora un periodismo que pretende desarrollar y divulgar el pensamiento crítico entre sus lectores.

Pese a que la revista presenta buenos resultados, como en la mayoría de los diarios analizados, Argia también muestra un decrecimiento de la calidad en el periodo 20012012. El promedio de la calidad entre 2001-2006 es de un 6,28 y en los últimos seis años analizados el promedio desciende a 6,19 .

\subsubsection{Argia, análisis sincrónico}

Excepto en el caso de Goiena y Le Monde, en el resto de las publicaciones analizadas incluida Argia, se aprecia la misma tendencia: la edición impresa posee mayor calidad que la edición digital. Lo cierto es que en el $31,82 \%$ de los textos que se han examinado en la edición digital se identifica algún error.

Argia $(1,24)$ es junto a El País $(1,41)$ quien manifiesta entre ambas ediciones mayor diferencia.En el caso de la revista vasca la justificación parece clara: debido a su periodicidad semanal, en la edición impresa disponen de más tiempo para seleccionar y elaborar las informaciones. En la edición digital por el contrario, funcionan como un diario que además debe responder a la inmediatez que exige la red. Lander Arbelaitz, redactor jefe del portal web de Argia, no duda en afirmar que la situación actual de incertidumbre es una puerta abierta a las oportunidades. Según el periodista, los medios pequeños y en concreto los que se editan en euskara, deben aprovechar las oportunidades que les brindan las nuevas tecnologías y utilizar bien los blogs, Youtube, Facebook o Twitter. Por otro lado, Arbelaitz aboga por emplear a la ciudadanía y a los movimientos sociales como fuentes de información. A través de esas prácticas, subraya Arbelaitz, los pequeños medios y en euskara deben propiciar un nuevo modo de consumo de información sin descuidar la calidad y ofreciendo un tratamiento lo más profesional posible. 


\subsection{Berria, análisis diacrónico}

El diario Berria no se publica los lunes pero el resto de los días de la semana tiene entre 48 y 52 páginas; al igual que Argia se difunde en la Comunidad Autónoma Vasca, Comunidad Foral de Navarra y País Vasco del Norte. De la muestra vasca, son los datos de este diario los que mejor se pueden equiparar a los resultados de la prensa europea, porque la ficha metodológica está diseñada para el estudio de los diarios de información general. En el siguiente gráfico se puede apreciar que Berria está 0,16 puntos por debajo del promedio de la prensa europea y únicamente Frankfurter Allgemeine Zeitung $(6,08)$ y El País $(5,47)$ se colocan por delante.

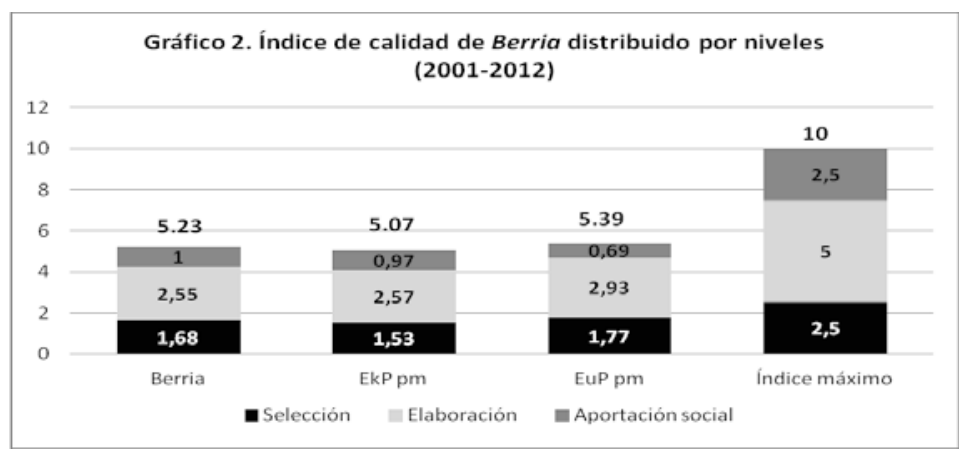

Fuente: Grupo de Investigación HGH

En el proceso de selección, Berria se sitúa por debajo de la media europea a una distancia de 8 centésimas. Entre las cinco variables que componen este apartado con una puntuación máxima de 0,50 cada una de ellas, la noticiabilidad se sitúa en 0,43 y la actualidad en 0,41 . El resto de los valores son más bajos aunque no se alejan demasiado de los estándares europeos.

En lo relacionado con el proceso de elaboración, la diferencia respecto a la media de la prensa de Europa es de 0,38 centésimas. En este apartado son seis las variables que se evalúan. En lo que respecta a la coherencia entre el titular y el desarrollo de los elementos de la noticia en el texto, el parámetro que hemos definido como Exactitud, obtiene un 0,92 . Sin embargo, en la profundización de los contenidos, no responde a todas y cada una de las clásicas preguntas del periodismo y se sitúa en 0,62. Asimismo, el estilo periodístico utilizado por Berria es adecuado, claro y conciso. Pese a que en general los valores logrados por el diario son buenos, el volumen de fallos en los elementos de titulación y en el texto, así como la poca elaboración en el apartado artístico hacen que la valoración final de esta apartado descienda.

Por el contrario, en lo que se refiere a la aportación social Berria está 0,31 centésimas por encima de la media europea y supera a Le Monde con 0,15 centésimas, que es el diario europeo más destacado en aportación social. Parece lógico que el diario dedique atención a este apartado, puesto que se trata de un proyecto creado con las aportaciones de la ciudadanía. Como viene ocurriendo desde que el Gobierno Vasco comenzara a subvencionar a los medios en euskara y pese a recibir ayudas de las instituciones públicas vascas, Berria se ha mantenido siempre al margen de los núcleos del poder y se puede interpretar que ello le ha servido para destacar en este apartado. 
Berria ha conseguido elevar su índice de calidad en los últimos años. El promedio de 2001-2006 es de 5,20 y en el segundo sexenio consigue incrementarlo hasta 5,26. La tendencia general entre los diarios analizados es descendente como ha quedado señalado anteriormente, salvo las excepciones de Frankfurter Allgemeine Zeitung y Le Monde.

No se puede obviar que durante el primer sexenio, concretamente el 20 de febrero de 2003 Euskaldunon Egunkaria fue clausurado por orden del juez Juan del Olmo, bajo la acusación de formar parte del "entramado de ETA". Hasta que el 21 de junio de ese mismo año se pusiera en marcha Berria se publicó Egunero, diario que también se ha analizado en esta investigación. El infortunio que supone un cierre de estas características y el esfuerzo económico y personal que requiere crear una nueva iniciativa desde cero y con las fuerzas mermadas, explica que a partir de 2003 en Berria se aprecie una clara tendencia a la baja que se mantiene hasta el 2008, año que refleja un notable incremento.

\subsubsection{Berria, análisis sincrónico}

Los datos del análisis sincrónico, al igual que en el resto de los diarios analizados, dejan en evidencia que la calidad de las ediciones impresas supera la calidad de las digitales. En el caso de Berria la distancia de 0,25 entre ambas ediciones es semejante a la que muestran los diarios europeos, excepto en el caso de El País y Argia cuya diferencia entre la edición impresa y la digital es mayor. En Goiena y Le Monde, como se explicará a lo largo de este artículo la tendencia es opuesta. Es decir, se observa una mayor calidad en la noticia de la edición digital que en la noticia de la edición impresa del día siguiente.

Martxelo Otamendi, director del diario Berria y ex director del clausurado Euskaldunon Egunkaria, subraya la doble crisis que sufren los medios editados en euskara: por un lado soportan la situación de la lengua minorizada y por otro la crisis económica, que desde siempre ha azotado a la prensa vasca. Según Otamendi, el hecho de pertenecer a una comunidad pequeña, no siempre es perjudicial, puesto que los receptores están muy identificados con los medios en euskara, pero eso no es razón para descuidar la calidad. De hecho, Otamendi incide en que "el futuro pasa por estar cerca del receptor y considera urgente y necesario un acuerdo entre los agentes del sector comunicativo vasco, la administración y los usuarios/receptores".

\subsection{Goiena, análi- sis diacrónico}

Como se puede apreciar en el gráfico, Goiena alcanza un valor inferior respecto a Argia y Berria. Las características pro-

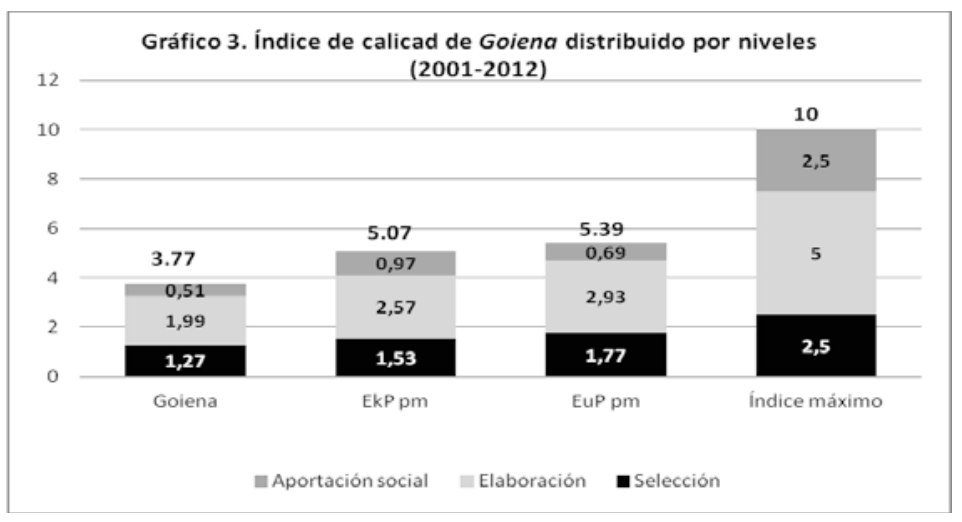

Fuente: Grupo de Investigación HGH 
pias de los medios de comunicación locales no han favorecido a Goiena ante una rigurosa metodología diseñada para evaluar la calidad de los diarios impresos de información general. Goiena se publica dos veces por semana (lunes y viernes) y abarca información local de la comarca guipuzcoana Debagoiena. De modo que en algunas de las variables utilizadas en esta investigación nunca podría lograr los valores de un diario de información general, debido a su carácter de publicación local no diaria. Por ello, la justificación de estos resultados responde a varios factores.

En esta primera parte del proceso deselección, en la variable que mide el carácter de las fuentes, Goiena obtiene un 0,11 puntos de un total de 0,5 . Utiliza fuentes identificadas con nombre y apellidos y con los cargos que corresponden, pero a menudo, aunque se intuye quién es la fuente, no aparece citada explícitamente. Puesto que se publica los lunes y los viernes, como ocurre en Argia, la actualidad consigue un valor de 0,24 de un total de 0,5 en la edición impresa. La variable que mide la noticiabilidad obtiene 0,43 puntos sobre 0,5 , lo que significa que la mayoría de las informaciones que selecciona Goiena para publicar en sus páginas son noticias que tienen consecuencias sociales, dejando de lado aquellos hechos que repercuten únicamente a un reducido grupo de personas y también los llamados pseudoeventos.

En cuanto al proceso de elaboración se contempla cierta debilidad en las variables que miden la profundidad y el número de perspectivas que integran las noticias. Son pocas las noticias que van más allá de las cinco clásicas preguntas del periodismo. Es decir, quedan sin respuesta las preguntas cómo y de dónde, dado que la mayor parte de las informaciones responden a las $4 \mathrm{o}$ en el mejor de los casos a las $5 \mathrm{~W}$-s. Es por eso que la variable de la profundidad obtiene un valor de 0,19 sobre 1 . Del mismo modo, en muy pocas ocasiones se introducen más de dos puntos de vista en las noticias. En la mayoría de las ocasiones se utiliza una única perspectiva argumentada, razón que esclarece la puntuación lograda, 0,21 de un 1 .

El estilo periodístico que utiliza Goiena es apropiado, claro y conciso. Así lo demuestra la puntuación de 0,93 otorgada en esta variable que tiene como valor máximo un 1. Asimismo, en ocasiones se aleja de las normas básicas del periodismo. En los elementos de titulación y sobre todo en los textos, tiende a utilizar la primera persona del plural. Si consideramos las normas básicas del periodismo, se debe evitar siempre el uso de la primera persona, salvo que aparezca entre comillas en forma de cita directa. Sin embargo, en los medios locales, dado el carácter de proximidad, se tiende a usar la primera persona del plural, error que cada vez es más frecuente en los medios audiovisuales pero que no por ello, es permisible y adecuado.

Goiena abusa del pretérito perfecto en los titulares. Es comprensible que a los y las periodistas les resulte difícil escribir en presente en la edición de los viernes sobre los hechos acontecidos dos o tres días antes, contraviniendo las normas del periodismo que indican que los titulares deben ir siempre en presente. También se han identificado repeticiones de vocablos en el titular y el subtítulo y errores de concordancia en las citas textuales. Todo ello ha incidido en que Goiena obtenga valores medios que se distancien del resto de las publicaciones en este apartado.

En el nivel de selección, al contrario que los otros dos medios vascos, Goiena ha conseguido valores más modestos. Las funciones de control al poder y el fomento del 
debate social apenas se reflejan en los ítems analizados en Goiena, dado que la mayoría de las noticias analizadas son descriptivas y carecen de profundidad porque los temas tratados no se prestan a ello. Sin embargo, cabe subrayar que la revista vasca garantiza la dignidad de las informaciones conseguidas y publicadas en la mayoría de las ocasiones, así que en esta variable ha conseguido un 0,48 sobre 0,5.

Como el resto de las publicaciones analizadas, Goiena presenta una tendencia descendente en su evolución. En sus primeros seis años la media de la calidad es de un 3,84 y en el segundo sexenio cae hasta 3,69.

\subsubsection{Goiena, análisis sincrónico}

Lo habitual, como se ha podido comprobar hasta el momento, es que la edición impresa de la noticia que ha servido de comparación a la de la edición digital logre una nota superior. Las dos únicas excepciones entre las publicaciones analizadas son $L e$ Monde y Goiena. Las razones de los resultados se deben fundamentalmente a la periodicidad bisemanal y a la muestra elegida para esta investigación. Por un lado, las noticias que se editan para la edición digital a menudo no se reflejan en la publicación del viernes, bien porque han perdido actualidad o bien porque de acuerdo a criterios profesionales se ha optado por no incluirlas. Por otro lado, porque para este estudio, y siguiendo las mismas pautas aplicadas al análisis diacrónico, se ha examinado la edición impresa del viernes. Por consiguiente, en la mayoría de los casos, aquellas noticias que en la edición digital contaban con un grado muy alto de actualidad, no se han desarrollado en la publicación impresa del viernes casi una semana más tarde.

Si consideramos la apuesta de Goiena por Internet y el modelo multimedia, no cabe duda de que existen diferencias entre la edición impresa y digital en algunas de las variables. A modo de ejemplo, la actualidad logra un 0,17 en la edición impresa y un 0,3 en la digital, ambas sobre un máximo de 0,5 . En cuanto a la utilización de elementos informativos complementarios, la edición impresa logra un 0,1 mientras que la edición digital obtiene un 0,2 , también ambas sobre 0,5 . Cabe citar que en la edición digital Goiena utiliza a menudo galerías de fotos y videos bien editados y apropiados para la noticia. En la edición digital se han registrado menos errores en los elementos de titulación y en el texto que en la edición impresa: el promedio de Internet es de $-0,05$ y el promedio de la edición impresa es de $-0,33$.

El responsable de gestión del Grupo de Comunicación Goiena tiene claro que uno de los retos de cara al futuro reside en mantener valores como la cercanía de la comunidad a la que va dirigida la publicación. También es consciente de que pese a la cercanía no se puede obviar la calidad, porque la competencia con publicaciones que trabajan en el idioma hegemónico es absolutamente descomunal. Uno de los objetivos del grupo de comunicación, según Arantzabal, es nombrar al ciudadano como "fuente". De hecho explica que tienen una experiencia piloto en marcha que está teniendo mayor aceptación que la prevista. La ciudadanía toma parte activa en la comunidad de Goiena, y aprecia la verdadera interactividad. El reto es, en palabras del responsable de gestión, hacer Goiena con la ciudadanía y para la ciudadanía. 


\subsection{Comparación de la prensa vasca con la prensa europea de referencia}

\subsubsection{Comparativa de los promedios}

La prensa vasca siempre aparece algo por debajo de la europea, pero no se puede obviar el nivel de difusión de los diarios europeos y vascos: Le Monde, el diario europeo de tirada más reducida de los que han sido objeto en esta investigación, tiene una difusión de casi 300.000 ejemplares, y la suma de Argia, Berria y Goiena difícilmente supera los 50.000 ejemplares. De modo que la diferencia de 0,32 entre los promedios no es extraordinaria si se tiene en cuenta que los medios vascos cuentan con muchos menos recursos económicos y personales.

La tendencia en ambos casos es descendente. La prensa vasca en su primer sexenio consigue una media de 5,11 y en el segundo logra un 5,04, un descenso de 0,07 puntos. La prensa europea, por el contrario, baja de 5,53 a 5,25, un descenso de 0,28 puntos. En consecuencia, la diferencia de la prensa europea sobre la prensa vasca a principios de la década, ha descendido en el segundo sexenio del periodo de análisis.

\subsection{2. Índices distribuidos por niveles}

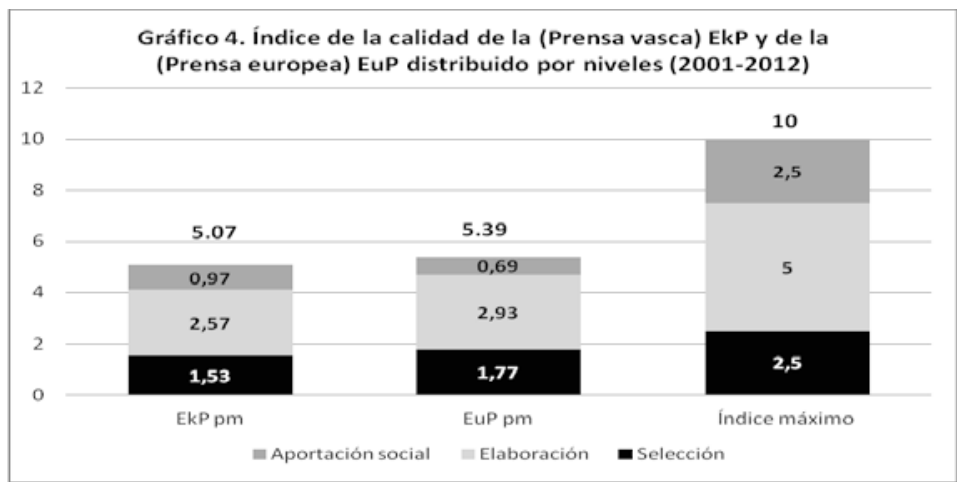

Fuente: Grupo de Investigación HGH

Como hemos señalado anteriormente, en los procesos de selección y elaboración la prensa europea sobresale respecto a la prensa vasca, en 0,24 y 0,37 puntos respectivamente.

Teniendo en cuenta las diferencias, los medios en euskara deberían mejorar su calidad en algunos aspectos que se detallan a continuación: deberían hacer un sobre-esfuerzo por reducir el número de errores que aparecen en los elementos de titulación y los textos, así como intentar profundizar más sus informaciones. Es decir, además de dar respuesta a las cinco clásicas preguntas del periodismo $(5 \mathrm{~W})$, deberían ir más allá y presentar las informaciones con antecedentes. En lo que se refiere a la profundización, el promedio de la prensa europea es de 0,63 y el de la prensa vasca de 0,54 sobre un valor máximo de 1 , pero ninguna de ellas recurre a los antecedentes para contextualizar las informaciones en la mayoría de las ocasiones. La prensa vasca debería poner más empeño en ofrecer más perspectivas en las informaciones publicadas, ya que de un valor máximo de 1, la prensa vasca se sitúa en 0,33 mientras que la prensa europea logra un 0,5 de media. 
No obstante, en el tercer índice que se refiere a la aportación social, la prensa vasca consigue mejores resultados que la prensa europea. Además, sobresale en todas y cada una de las variables que componen este nivel. Se puede intuir que el hecho de ser pequeño y modesto concede mayor capacidad para llegar a las informaciones de un modo más cercano, no solo en el caso de Goiena, que es una publicación local, también en el caso de Argia y Berria, que se dirigen a una comunidad lingüística más reducida y ello les permite desarrollar con más facilidad las variables que componen el apartado de la aportación social. Además, la cercanía, exige mayor sensibilidad hacia ciertos temas, como son entre otros, respeto a la dignidad humana, posicionarse a favor de la diversidad cultural o mostrar actitudes que rechacen la discriminación de los grupos sociales menos favorecidos.

\subsubsection{Diversidad}

En la prensa europea el protagonista principal de las informaciones es el gobierno en el $28 \%$ de los casos. Financial Times es la excepción, ya que en su caso los protagonistas por excelencia son los agentes económicos, tal como cabía esperar. El protagonismo que la prensa vasca otorga al gobierno es mucho menor como se puede apreciar en el porcentaje del 10\% obtenido. Además en los tres medios vascos analizados destacan otros protagonistas como agentes sociales, culturales y económicos, entre otros. Este resultado, junto con la variable del control al poder manifiesta que la prensa vasca toma más distancia respecto al poder que la prensa europea. Una de las razones de ese alejamiento puede responder a que el euskara y los medios editados en euskara se encuentran fuera de los núcleos del poder.

En el apartado de la diversidad otras dos variables importantes son las que corresponden al género del/la protagonista y la presencia de los colectivos marginados. Los valores de todas las publicaciones son muy bajos, sobre todo en lo que se refiere a la presencia de la mujer, aunque la prensa vasca está por delante de la prensa europea. En el 6,5\% de las informaciones de la prensa vasca la mujer es protagonista y en el $8,9 \%$ de las informaciones lo es algún colectivo marginado. En la prensa europea en cambio, la mujer es protagonista en el $4,2 \%$ de las informaciones y los colectivos marginados en el $6,15 \%$ de los textos.

\subsubsection{El salto a internet}

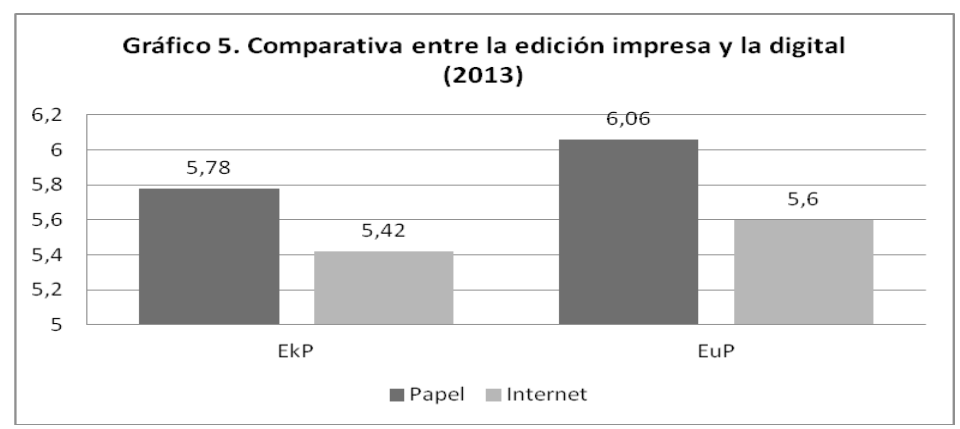

Fuente: Grupo de Investigación HGH 
Los resultados obtenidos dejan en evidencia que tanto en la red como en la edición impresa la prensa europea sobresale respecto a la prensa vasca. Sin embargo hay un dato que llama la atención: la diferencia entre el papel y la red en la prensa vasca es de 0,36 y en la prensa europea es de 0,46 . A priori, podría resultar lógico que la prensa vasca tuviese más dificultades para enfrentarse a las nuevas tecnologías, debido a la escasez de recursos, pero según los datos cabe esta otra interpretación: los medios vascos han tenido dificultades similares a las de los medios europeos para implantar las nuevas tecnologías en sus redacciones y pese a su modestia, han respondido bastante bien.

En este sentido, resultan interesantes las opiniones de los lectores de la prensa digital en euskara recogidas en un estudio sobre la calidad de las ediciones digitales de los medios de comunicación en Internet realizado por la empresa CIES para el equipo HGH. Los lectores y lectoras de la prensa diaria en euskara (Berria) consideran que la calidad de la edición digital de Berria es muy buena (11\%), es buena $(67 \%)$, es regular (12\%), es mala (>9\%) y es muy mala, no sabe o no puede valorar $(0 \%)$.

Berria y Gara (este último, un proyecto social publicado mayoritariamente en castellano) son los dos diarios que mayor porcentaje de lectores y lectoras tienen dispuestos a pagar por los contenidos en Internet. Si sumamos los porcentajes de las personas dispuestas a pagar y las que sí lo harían dependiendo del importe, Berria y Gara logran los mejores resultados con un 33\% y un 30\% respectivamente. Los datos demuestran el nivel de apego, adhesión y compromiso de sus lectores con ambos proyectos.

\section{Conclusiones}

Los medios vascos que por tradición y por tamaño son pequeños, tomados en su conjunto, superan los parámetros estándares de calidad. La rigurosa metodología aplicada le otorga a la prensa vasca una nota media de 5,07 en una escala de 10. En el mismo periodo de tiempo (2001-2012) los medios europeos de referencia, con mayor tradición y mayor dimensión, obtienen una media de 5,39.

El índice de calidad de la prensa vasca como el de la prensa europea muestran un decrecimiento en el periodo 2001-2012. Durante toda esa etapa la calidad de la prensa vasca ha descendido 20 centésimas mientras que el decrecimiento de la prensa europea ha sido de 28 centésimas. Los valores son significativos puesto que los márgenes que establece el método son muy ajustados y rigurosos. En ese sentido existen motivos de preocupación, sobre todo porque no se atisban signos para cambiar la tendencia descendente, en ninguno de los dos casos.

La comparativa entre la edición digital y la edición impresa en 2013 de las informaciones en euskara, deja en evidencia que las noticias de la edición impresa están mejor elaboradas que las que se publican en la red. En las ediciones europeas la tendencia es semejante. Por lo tanto, se puede concluir que el ritmo frenético que impone Internet perjudica la calidad de la información. La inmediatez provoca un periodismo caracterizado por la prisa y con ello, la rapidez y las probabilidades para cometer errores aumentan considerablemente. Parece difícil hacer las cosas rápido y bien, tan difícil como que confluyan la inmediatez y la profundidad.

En los resultados del índice de calidad de los medios vascos examinados hay diferencias por las características propias de cada medio. La metodología empleada ha 
favorecido a algunos y ha perjudicado a otros. Argia ha obtenido un valor medio de 6,2.La periodicidad semanal le concede la posibilidad de elaborar los textos con más profundidad, además, en su caso solo se han analizado las noticias de gran formato y eso le ha favorecido notablemente. El índice de calidad logrado por el diario Berria es de 5,23. En este caso, el método se ajusta perfectamente a las características del periódico. Berria se coloca entre los valores alcanzados por Le Monde $(5,16)$ y El País $(5,47)$. A pesar de que los parámetros de calidad son equiparables, las diferencias entre los tres diarios citados son notables en lo que se refiere a la tradición, al volumen, a la difusión, y a la capacidad económica de cada uno de ellos. El caso de Goiena, una publicación bisemanal y de carácter local, es especial. Logra un resultado más modesto $(3,77)$ y como ha quedado señalado anteriormente, el método empleado no le ha beneficiado. Es líder de audiencia en la comarca gipuzkoana del Alto Deba, se reparte los viernes gratuitamente, pero sería conveniente que cuidara más la calidad con la finalidad de corregir los errores que se han identificado, sobre todo los relacionados con las normas básicas del periodismo.

Los tres medios en euskara analizados en este trabajo tienen una característica común que no comparten con los diarios europeos. Las tres publicaciones vascas son fruto de iniciativas sociales, que hace que estén muy cerca de lo que internacionalmente se conoce como modelos de community media. Aunque sus recursos son escasos, la capacidad para llegar al receptor es enorme. Publicadas en una lengua minorizada y pertenecientes a una comunidad sin Estado, la opción de estas publicaciones para el desarrollo de la nación es muy importante y así ha quedado reflejado en esta investigación: estos medios tienen una gran capacidad para cohesionar la sociedad vasca y sobre todo la comunidad de vascohablantes. Prueba de ello es que en la encuesta realizada por CIES, Berria es el diario que más porcentaje de lectores tiene dispuestos a pagar por la información en la red.

Si bien en los procesos de selección y de elaboración los medios vascos se sitúan por debajo de los diarios europeos, no sucede lo mismo en el nivel de aportación social. En ese apartado la prensa vasca se sitúa 28 centésimas por encima de la prensa referencia europea, lo que demuestra una mayor sensibilidad para ejercer el control sobre el poder, para promover el debate social, para garantizar la dignidad de las personas, para mostrar la diversidad cultural y para combatir la exclusión social. En este caso podemos afirmar que tanto los medios más grandes como los más pequeños pueden enseñar y aprender unos de otros.

\section{Referencias bibliográficas}

ARANTZABAL, Iban (2013): "Retos de los medios audiovisuales en lenguas minorizadas". Mesa Redonda. En Primeras Jornadas Internacionales sobre el Futuro del Periodismo de Calidad. Facultad de Ciencias Sociales y de la Comunicación de Leioa (Bizkaia), Grupo HGH. En: http://futurodelperiodismo.wordpress.com/

ARBELAITZ, Lander (2013): "Fortalezas y debilidades del nuevo escenario mediático". Mesa Redonda. En Primeras Jornadas Internacionales sobre el Futuro del Periodismo de Calidad. Facultad de Ciencias Sociales y de la Comunicación de Leioa (Bizkaia), Grupo HGH. En: http://futurodelperiodismo.wordpress.com/ 
BUCHER, Hans-Jürgen; ALTMEPPEN, Klauss-Dieter (ed.) (2003): Qualität im Journalismus: Grundlagen, Dimensionen, Praxismodelle. Wiesbaden, Westdeutscher Verlag.

CIES (2013). Estudio cuantitativo y cualitativo sobre las ediciones electrónicas de la prensa europea de calidad. Realizado para la Universidad del País Vasco/Euskal Herriko Unibertsitatea.

DÍAZ NOCI, Javier (2012): Historia del periodismo vasco (1600-2010). Donostia, Mediatika. Cuadernos de Medios de Comunicación-Eusko Ikaskuntza, Nº13.

GÓMEZ MOMPART, Josep Lluís y PALAU, Dolors (2013): "El reto de la excelencia. Indicadores para medir calidad periodística", en GÓMEZ MOMPART, Josep Lluís; GUTIERREZ, Juan Francisco; PALAU, Dolors (2013): La calidad periodística. Teorías, investigaciones y sugerencias profesionales, Barcelona, Servei de Publicacions de la Universitat Autònoma de Barcelona, pp.17-38.

GONZALEZ GOROSARRI, Maria (2011): Albisteen Kalitatea (Research on Basque Media's News Quality). Leioa, University of the Basque Country.

MAURER, Torsten (2005): Fernsehnachrichten und Nachrichtenqualität: Eine Längsschnittstudie zur Nachrichtenentwicklung in Deutschland. München, Reinhard Fischer.

MCQUAIL, Denis (1992): Media performance. Mass communication and the public interest.London, Sage.

OTAMENDI, Martxelo (2013): "Retos de la prensa y radio en lenguas minorizadas". Mesa Redonda. En Primeras Jornadas Internacionales sobre el Futuro del Periodismo de Calidad. Facultad de Ciencias Sociales y de la Comunicación de Leioa (Bizkaia), Grupo HGH. En: http://futurodelperiodismo.wordpress.com/

RAMIREZ DE LA PISCINA, Txema (2014): "Periodismo de calidad en tiempos de crisis: Un análisis de la evolución de la prensa europea de referencia (2001-2012)", Revista Latina de Comunicación Social, no 69.

VEHLOW, Bernd (2006): Qualität von Spätnachrichten-Sendungen.München, Reinhard Fischer.

VV.AA. (2005): Kazetaritza euskaraz: oraina eta geroa. Bilbao, Servicio Editorial Universidad del País Vasco.

VV.AA. (2013): Euskal Hedabideen Etxea, tokikoen ikuspegitik. Tokikom, Aretxabaleta.

WEISCHENBERG, Siegfried; LOOSEN, Wiebke BEUTHNER, Michael (ed.) (2006): Medien-Qualitäten: Öffentliche Kommunikation zwischen ökonomischem Kalkül und Sozialverantwortung. Konstanz, UVK.

ZABALETA, Iñaki (1997): Komunikazioaren eta ikerkuntzako metodologia. Bilbo, Udako Euskal Unibersitatea.

ZABALETA, Iñaki et al. (2014): "Realidad y efectos de la doble crisis en los medios en lengua vasca, gallega y catalana", Libro de Comunicaciones del IV Congreso AE-IC Espacios de Comunicación. Bilbao, pp. 105-111. 